\title{
BellaGel breast implant: 6-Year results of a prospective cohort study
}

Joon Seok Oh ${ }^{1}$, Jae Hoon Jeong ${ }^{1}$, Yujin Myung ${ }^{1}$, Jeongseok $\mathrm{Oh}^{1}$, Shin Hyeok Kang ${ }^{1}$, Eonju Park ${ }^{1}$, Ara Kim², Sa Ik Bang ${ }^{2}$, Chan Yeong Heo ${ }^{1}$

${ }^{1}$ Department of Plastic and Reconstructive Surgery, Seoul National University Bundang Hospital, Seoul National University College of Medicine, Seongnam; ${ }^{2}$ Department of Plastic and Reconstructive Surgery, Samsung Medical Center, Sungkyunkwan University School of Medicine, Seoul, Korea

Background This is the first clinical study conducted among Asian women using breast implants manufactured by an Asian company. Four-year data regarding the safety and efficacy of BellaGel breast implants have already been published, and we now report 6-year data. Methods This study was designed to take place over 10 years. It included 103 patients who underwent breast reconstruction or augmentation using BellaGel breast implants. The rates of implant rupture and capsular contracture were measured and analyzed to evaluate the effectiveness of the breast implant.

Results At patients' 6-year postoperative visits, the implant rupture and capsular contracture rates were $1.15 \%$ and $2.30 \%$, respectively. The implant rupture rate was $3.77 \%$ among reconstruction cases and 0\% among augmentation cases. The capsular contracture rate was 5.66\% among reconstruction cases and $0.83 \%$ among augmentation cases.

Conclusions The 6-year data from this planned 10-year study suggest that the BellaGel cohesive silicone gel-filled breast implant is an effective and safe medical device that can be used in breast reconstruction and augmentation.

Keywords Breast implant / Mammaplasty / Capsular contracture

\author{
Correspondence: \\ Chan Yeong Heo \\ Department of Plastic and \\ Reconstructive Surgery, Seoul \\ National University Bundang Hospital, \\ Seoul National University College of \\ Medicine, 82 Gumi-ro 173beon-gil, \\ Bundang-gu, Seongnam 13620, Korea \\ Tel: +82-31-787-7229 \\ Fax: +82-31-787-4055 \\ E-mail: lionheo@snu.ac.kr \\ Sa lk Bang \\ Department of Plastic and \\ Reconstructive Surgery, Samsung \\ Medical Center, Sungkyunkwan \\ University School of Medicine, \\ 81 Irwon-ro, Gangnam-gu, Seoul \\ 06351, Korea \\ Tel: $+82-2-3410-2235$ \\ Fax: +82-2-3410-0036 \\ E-mail: si55.bang@samsung.com
}

Hans Biomed provided financial support for this study.

\section{INTRODUCTION}

For many women, breasts have a symbolic meaning and contribute to self-esteem and satisfaction. Extremely small breasts may not only have a negative psychological impact, but also can be associated with relationship-related conflict. As such, many healthy people with small breasts who desire larger ones have used massage or hormone therapy in the past. However, these methods often failed, leading to an increase in the number of patients receiving augmentation mammoplasty since the 1990s. Breast augmentation was the most common cosmetic surgical procedure performed in the United States in 2018, with a total of 313,000 procedures, representing a $4 \%$ increase compared to 2017 [1]. In the past, the perception existed that only celebrities 
and people with psychological issues underwent cosmetic plastic surgery. The Confucian tenet of avoiding artificial changes to one's inherited body, which prevailed in the past, has also contributed to a negative stigma toward plastic surgery. However, demand for and positive attitudes towards plastic surgery are burgeoning due to increased incomes spurred by economic growth, changes in ethical values, and the development of broadcast media. According to an international survey on aesthetic/cosmetic procedures that were performed in 2018, breast augmentation is the most common cosmetic procedure worldwide. A total of 1,862,506 breast augmentation procedures were performed in 2018, which constituted an increase of $6.1 \%$ compared to 2017 [2].

In 1963, Cronin and Gerow sparked a revolution in breast augmentation by developing silicone [poly(dimethylsiloxane)] implants [3]. Domestically, no clear evidence of silicone-associated risk has been found despite continuous research after the suspension of the Dow Corning artificial silicone breast implant. In 1992, Dow Corning implants were introduced as second-generation implants. They contained a high-liquidity gel that increased the risk of the systemic spread of silicone after implant rupture. Third-generation cohesive gel implants overcame this disadvantage by using the high cohesiveness of the gel to delay the systemic diffusion of silicone after implant rupture [4]. Hans Biomed has developed breast implants with improved safety by using such a cohesive gel.

Hans Biomed is attempting to contribute to the development of the domestic medical device industry by confirming the safety and high quality of their cohesive silicone gel-filled breast implant (CoSBI). This is the first clinical study conducted among Asian women using breast implants manufactured by an Asian company, and it is expected to continue for 10 years. Four-year data regarding BellaGel breast implants were previously published, and we now report 6-year interim data from the 10-year study.

\section{METHOD}

The Ministry of Food and Drug Safety (MFDS) approved the instructions and consent forms for this study. All aspects of the study were approved by the MFDS or the institutional review board (IRB) independent of the institution that conducted the study (Seoul National University Bundang Hospital IRB No. E-100/049-001 and Samsung Medical Center IRB No. SMC 2008-09-073). The study adhered to the International Conference on Harmonization guidelines and the Declaration of Helsinki. The study was conducted according to the Korean Good Clinical Practice guidelines.

\section{Study design}

The study was conducted to test the safety and efficacy of the Hans Biomed CoSBI used for breast reconstruction (due to cancer, trauma, or congenital defects) or augmentation mammoplasty in patients aged 22 years or older. Patients requiring contralateral breast augmentation after reconstruction were considered to be in the reconstruction category. The study examined the rupture and capsular contracture rates at postoperative year 2 . In addition, the occurrence of connective tissue disease, changes in breast size, and the incidence of capsular contracture and rupture were measured at each visit. Other complications, adverse events, laboratory abnormalities, vital signs, nipple sensitivity, and physical examination results were also evaluated and recorded if clinically significant. Any adverse events were recorded along with the severity and the causal relationship with the implant. The study was conducted from $\mathrm{Au}$ gust 24, 2010 to November 2, 2018. The inclusion and exclusion criteria are outlined in Table 1.

Patient screening included general laboratory testing, a chest $\mathrm{X}$-ray, an electrocardiogram for preoperative assessment, mammography, a test of nipple sensitivity, a medical photo, and a survey. The implant was inserted within 30 days after screening. Patient safety and satisfaction were evaluated at postoperative months 1, 3, 6, 12, and 24. Patient satisfaction was measured using the Rosenberg self-esteem scale, the Body Esteem Scale, and the 36-item Short Form (SF-36) health survey at every visit. Follow-up was conducted at postoperative years 2,4 , and 6 , and

Table 1. Inclusion and exclusion criteria

\begin{tabular}{|c|c|}
\hline Inclusion criteria & $\begin{array}{l}\text { - Female; at least } 22 \text { years old } \\
\text { - Requesting bilateral augmentation mammoplasty or } \\
\text { - Requiring breast reconstruction for correction of breast } \\
\text { deformity due to mastectomy, trauma, or congenital defect } \\
\text { - Fertile; tested for pregnancy with a negative result } \\
\text { - Agreed to participate in the study and provided written } \\
\text { consent } \\
\text { - Willing to follow the study instructions }\end{array}$ \\
\hline Exclusion criteria & $\begin{array}{l}\text { - History of previous breast reconstruction of augmentation } \\
\text { mammoplasty } \\
\text { - Autoimmune disease (systemic lupus erythematosus, } \\
\text { chronic glomerulonephritis, or rheumatic arthritis) } \\
\text { - Uncontrolled active infectious disease } \\
\text { - Unable to insert breast implant due to soft tissue defect or } \\
\text { history of radiotherapy } \\
\text { - Systemic cancer, relapse of breast cancer, or any cancer } \\
\text { not receiving appropriate treatment } \\
\text { - Keloid skin } \\
\text { - Ineligible for general anesthesia } \\
\text { - Ineligible for magnetic resonance imaging due to } \\
\text { claustrophobia or metal inside body } \\
\text { - History of alcohol, drug abuse, or addiction to plastic } \\
\text { surgery that may affect compliance with study protocol } \\
\text { - Ineligible for study as determined by investigator }\end{array}$ \\
\hline
\end{tabular}


follow-up is also planned at years 8 and 10 .

Nipple sensitivity was evaluated using Semmes-Weinstein monofilaments. The diameter of the smallest filament that the patient could feel was set as the pressure threshold. The sensitivity was tested at the nipple and the upper, lower, medial, and lateral areola, and the average was determined. Nipple sensitivity was measured preoperatively and at every postoperative visit, which took place at postoperative months $1,3,6$, and 12 and years 2,4 , and 6 .

The patient data were divided into three groups: the safety set, the intention-to-test (ITT) set, and the per-protocol (PP) set. The safety set and the ITT set included any patient who received at least one operation. The PP set included patients who received at least one operation and all evaluations expected up to postoperative year 2 . The ITT set consisted of the main population; an additional evaluation was performed in the PP set, and data regarding safety were evaluated based on the safety set. Mean values were provided for continuous variables and frequencies were provided for categorical variables. This clinical study was intended to continue for a total of 10 years, and this publication includes patients' results through postoperative year 6 . The primary goal of the study was to confirm whether the incidence of implant rupture was below $1 \%$ and the incidence of capsular contracture was below $5 \%$, with the upper limit of the $95 \%$ confidence interval being smaller than $4 \%$. Implant rupture was assessed using breast magnetic resonance imaging at postoperative months 6, 12, and 24 and then at 2-year intervals. Cases of implant rupture due to traffic accidents or trauma due to patient carelessness were excluded. If the incidence of implant rupture was below $1 \%$ and the upper limit of the $95 \%$ confidence interval was smaller than $4 \%$, the implant could be considered safe from rupture risk. The Baker classification scale was used to evaluate capsular contracture. Baker grade III and IV patients were diagnosed with capsular contracture. If the incidence of capsular contracture was below 5\% and the upper limit of the $95 \%$ confidence interval was smaller than $10 \%$, the implant could be considered safe from capsular contracture.

Capsular contracture data were analyzed using Kaplan-Meier survival analysis. The paired t-test was used to detect statistically significant changes in the Rosenberg self-esteem scale, the Body Esteem Scale, the SF-36 scale, and breast circumference.

Adverse events after implant insertion were defined as treatment-emergent adverse events. Any adverse events were standardized using the Medical Dictionary for Regulatory Activities. The incidence of adverse events was classified using System Organ Class and Preferred Term classification. For each adverse event, the causal relationship with the implant as well as the severity, result, and treatment were provided. Patients were dropped from the trial upon their request or when any adverse event or complication made it impossible to continue the trial.

\section{Surgical method}

The incisions for breast reconstruction and augmentation mammoplasty include transaxillary, inframammary fold, and periareolar incisions. The implants were inserted in the subglandular, subfascial, or submuscular layer. The type of incision and the insertion layer were determined based on the clinical decision of the operator.

For augmentation mammoplasty, patients were laid in the supine position under general anesthesia with plain tube insertion. Typical breast draping was performed. The operation table was maneuvered to orient the patient in a sitting position so that 4-cm incision lines could be drawn in the axillary crease, inframammary fold, or periareolar areas depending on incision type. A sizer was used to select the implant size. Tisseel spray was applied, and an implant irrigated with normal saline was inserted. Hemovac drains were inserted into both breasts. After wound closure, a downward compressive dressing was applied.

For tissue expander insertion, the sternal notch, the inframammary fold, and the midline of the sternal notch and xyphoid process were designed to be in the upright position. After mastectomy was performed by general surgeons, meticulous hemostasis and normal saline irrigation were performed. The pectoralis muscle was identified and elevated along the submuscular plane. The pocket for the tissue expander was dissected; the expander was inserted into the subpectoral plane, and the pectoralis muscle was draped to cover the expander. The lower border of the pectoralis major muscle was covered using acellular dermal matrix, such as Cryoderm, Dermacell, Megaderm, or Alloderm. Data regarding the size or type of acellular dermal matrix were not collected. Expanders of unspecified type were inserted in the dual plane, a Hemovac drain was inserted into the subpectoral pocket and subcutaneous plane, and the incision was primarily closed. Tissue expansion with normal saline was performed in a manner that avoided compromising skin envelope viability. A compressive dressing was then applied. Tissue expansion was performed once every 1 or 2 weeks for 3 months in an outpatient clinic. No direct-to-implant cases were present in the breast reconstruction group.

Tissue expander removal and implant insertion were performed 3 to 6 months after tissue expander insertion. An incision was made along the previous scar. The saline in the tissue expander was drained before the expander was removed. Dissection was performed to modify the inframammary fold position, pocket location, and size, and the pocket was irrigated. A Hemovac drain was inserted in the subpectoral pocket, and the 
implant was then inserted. The wound was closed layer by layer, and a compressive dressing was applied.

Postoperative antibiotics (cefixime, cefdinir, and cefalexin), analgesics (aceclofenac and Carol-F ibuprofen/arginine tablets), and Beszyme were prescribed to inhibit bacterial growth and prevent inflammation. From postoperative day 3 or 4 to postoperative 6 months, breast massages were done two to three times per day for at least 10 minutes to prevent capsular contracture.

\section{RESULTS}

The current study evaluated the safety of the Hans Biomed CoSBI, a third-generation breast implant composed of cohesive silicone gel. This study presents the results of the first 6 years of a 10-year clinical study. A total of 164 patients were enrolled in the study, with 61 patients excluded and 103 patients meeting the inclusion criteria. A total of 51 patients underwent reconstruction (54 cases, including bilateral procedures), and $52 \mathrm{pa}-$ tients underwent augmentation (123 cases, including bilateral procedures). All patients who used the product were included in the ITT and safety sets. Eight cases of reconstruction (seven patients) and 20 of augmentation (10 patients) were dropped from the study.

In the second year, 54 cases of reconstruction and 123 cases of augmentation were included in the ITT set. A total of 31 reconstruction cases (57.41\%) and 58 augmentation cases (47.15\%) were included in the PP set. The PP set was revised every 2 years, mostly due to patient drop-off from the study due to patients' request.

In the 4th year, the ITT and safety sets still consisted of 54 reconstruction and 123 augmentation cases. At this point, 12 reconstruction and 28 augmentation cases were included in the PP set.

In the 6th year, again, 54 reconstruction and 123 augmentation cases were included in the ITT and safety sets. The PP set in the 6 th year included six cases.

The 6-year implant rupture rates in the ITT and PP sets exceeded $1 \%$, but the capsular contracture rate was below $5 \%$, and the upper limit of the $95 \%$ confidence interval was below $10 \%$. The incidence of connective tissue disease in the ITT set was two cases at 3 months and three cases by year 6 . No cases of connective tissue disease were observed in the PP set. Breast size showed no significant difference until the 6 th year. All patients in the ITT and PP sets were satisfied after surgery. In the ITT set, among patients who underwent reconstruction, the median value of the Rosenberg self-esteem scale was 30 preoperatively and 29 at postoperative year 6 .

Patients' average age was $44.67 \pm 7.78$ years for reconstruction
Fig. 1. Proportion of patients with decreased nipple sensitivity

Recovery of nipple sensitivity was observed over time.

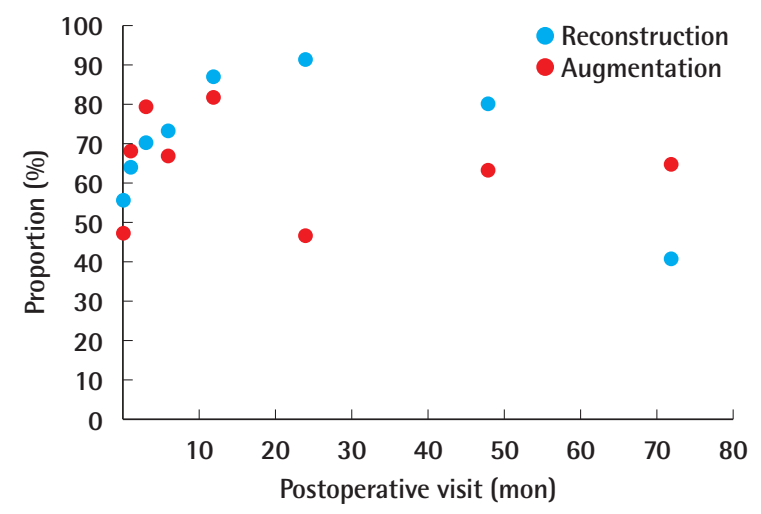

cases and $28.73 \pm 5.61$ years for augmentation cases. The average height was $158.48 \pm 4.59 \mathrm{~cm}$ for reconstruction cases and $161.72 \pm 4.45 \mathrm{~cm}$ for augmentation cases, and the average weight was $54.98 \pm 6.53 \mathrm{~kg}$ for reconstruction cases and $50.94 \pm$ $4.87 \mathrm{~kg}$ for augmentation cases. The reconstruction cases did not include any smokers, whereas 14 of the patients (26.92\%) who underwent augmentation were smokers.

Of the safety set of 103 patients, 94 (91.26\%) experienced at least one adverse event. Thirty (29.13\%) were adverse device events, and 29 (28.16\%) were significant adverse events. Eight cases $(7.77 \%)$ involved significant adverse device events (SADE), including three cases of implant-associated capsular contracture, one case of deep vein thrombosis, one case of stress cardiomyopathy, one case of breast dysplasia, and two cases of device breakage. All patients who experienced SADE recovered, and one patient with recurrent breast cancer is currently recovering. The most frequent adverse event was procedural pain $(60.78 \%)$. The proportion of patients with a decrease in nipple sensitivity was also evaluated, and the results are presented in Fig. 1.

At year 6, the proportion of patients with decreased nipple sensitivity decreased among reconstruction cases and increased among augmentation cases.

No case of implant rupture occurred during the first 4 years. At the 6-year visit, among the ITT group, implant rupture had occurred in two out of 26 cases $(7.69 \%)$ in the reconstruction group and in no cases in the augmentation group. The upper limit of the $95 \%$ confidence interval was $8 \%$. Cumulatively, the total number of cases with implant rupture among the reconstruction group was two of 53 (3.77\%), whereas no cases of implant rupture were noted among the augmentation group. Across both reconstruction and augmentation, implant rupture occurred in two of 174 cases (1.15\%), and the upper limit of the 
Fig. 2. Implant rupture at each postoperative visit

No cases of implant rupture were observed in augmentation patients. Two cases of implant rupture were observed in reconstruction cases.

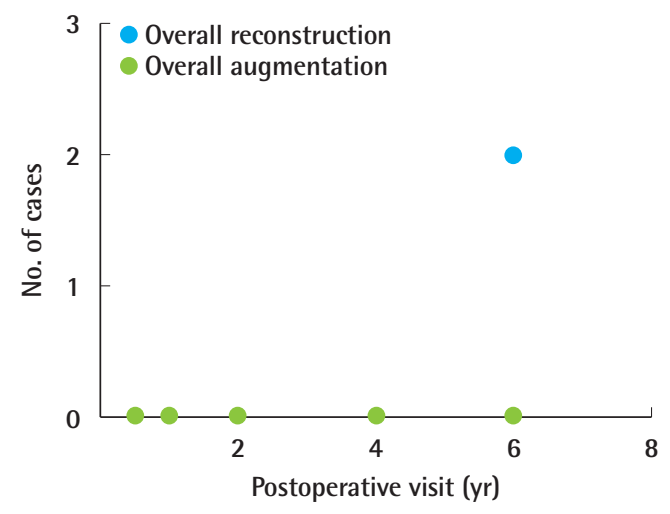

95\% confidence interval was 3\%. The implant rupture rate at each visit is presented in Fig. 2.

No case of capsular contracture was observed prior to postoperative 6 months. At postoperative 12 months, one of 53 cases (1.89\%) demonstrated capsular contracture. The upper limit of the $95 \%$ confidence interval was $2 \%$. In the reconstruction group, four cases of capsular contracture among 51 cases (7.84\%) were documented at postoperative 24 months, whereas no cases were observed in the augmentation group. At postoperative 48 months, two of 29 cases (6.90\%) demonstrated capsular contracture in the reconstruction group and one out of $69(1.47 \%)$ demonstrated capsular contracture in the augmentation group at postoperative 48 months. The upper limit of the confidence interval was $7 \%$. At postoperative year 6 , one out of 30 cases $(3.33 \%)$ demonstrated capsular contracture among the reconstruction patients and no cases demonstrated capsular contracture among augmentation patients. The upper limit of the confidence interval was 0.05 at postoperative year 6 . The capsular contracture rate at each visit is outlined in Fig. 3.

At postoperative 3 months, two patients displayed symptoms of connective tissue disorders. Both were augmentation cases, with patients who were suffering from urticaria. In total, three patients with symptoms of connective tissue disorders were noted at postoperative 6 years, all of whom had undergone reconstruction. The specific symptoms were alopecia (two patients) and urticaria (one patient).

The changes in upper chest circumference, lower chest circumference, and breast circumference after 1 month were $-0.33 \pm$ $2.69 \mathrm{~cm},-0.31 \pm 2.19 \mathrm{~cm}$, and $-1.49 \pm 2.42 \mathrm{~cm}$, respectively, for reconstruction cases and $4.05 \pm 2.83 \mathrm{~cm},-0.5 \pm 2.43 \mathrm{~cm}$, and $3.17 \pm 2.19 \mathrm{~cm}$, respectively, for augmentation cases. The change
Fig. 3. Capsular contracture at each postoperative visit

More cases of capsular contracture were observed among reconstruction cases.

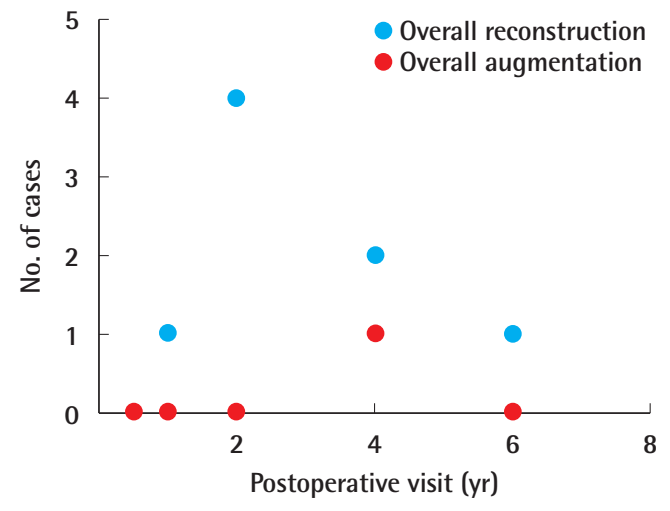

in values was less than $1 \mathrm{~cm}$ until postoperative year 2 , while the change was greater than $1 \mathrm{~cm}$ in postoperative years 4 and 6 . Breast circumference decreased continuously from postoperative 1 month to 6 years.

The median value of the Rosenberg self-esteem scale was above 30 points for reconstruction and augmentation at each visit. For the SF-36 health survey, the median score was above 101 points at every visit for both reconstruction and augmentation. Among reconstruction cases, median body esteem scales were above 105 points preoperatively and at postoperative 6,12 , and 24 months. At postoperative 4 years, the median score was 115 points, and at postoperative 6 years, it was 116 points. In augmentation cases, the median Body Esteem Score was 116 points preoperatively. However, that score decreased to 113 and 112 points at postoperative 6 and 12 months, respectively, and it increased again to 119 and 127 points at postoperative years 2 and 4 , respectively.

\section{DISCUSSION}

The study evaluated the CoSBI, a third-generation breast implant manufactured using cohesive silicone gel. The improved cohesiveness delays the diffusion of silicone after rupture of the implant. Hans Biomed developed a breast implant with enhanced safety by using such a cohesive silicone gel. The goal of this study was to evaluate the efficacy and safety of the newly developed implant. The clinical trial lasted from August 24, 2010 to November 2, 2018. The most important factors in using breast implants in reconstruction and augmentation are patient satisfaction and the minimization of breast implant-related complications. Implant rupture, capsular contracture, implant 
leakage, and infection are common complications of breast implants. Cohesive silicone gel is composed of cross-linked polymers. The increase in cohesiveness of the gel reduces spilling of the gel. This leads to decreased rates of capsular contracture, implant rupture, and leakage. With this reduction in the risk of complications, the use of breast implants in augmentation mammoplasty is increasing.

The BellaGel breast implant is the first breast implant manufactured by an Asian company. The product meets the requirements outlined by the American Society for Testing and Materials, and it has passed various tests of silicone gel-filled breast implants [5]. The BellaGel breast implant is manufactured in a wide range of implant volumes at a given implant width, allowing better fit of various breast shapes and sizes. Certification by the European Community and the International Organization for Standardization 13485:2003 (certificate No. FM581168) confirm the safety of BellaGel breast implant products.

The results of this 6-year clinical study show the safety and effectiveness of BellaGel breast implants. At patients' 6-year postoperative visits, the implant rupture and capsular contracture rates were $1.15 \%$ and $2.30 \%$, respectively. This is comparable to the rates associated with other breast implants [6-9].

The rupture rate was $0.5 \%$ among augmentation and $0.9 \%$ among reconstruction cases in a 3-year cohort study conducted with Mentor MemoryGel implants [7]. The rupture rate was $0.7 \%$ in augmentation and $1.3 \%$ in reconstruction for Allergan Style 410 implants in a 3-year study [8], and the rupture rate was $1.8 \%$ in Silimed high-strength silicone gel implants in a 5-year study [9]. The rupture rate of the BellaGel breast implant at 6 years was $1.15 \%$, indicating that its safety is comparable to that of other implants. With its relatively thick shell and high tensile strength, BellaGel breast implants carry a minimal risk of silicone leak.

The capsular contracture rate was $0.83 \%$ in augmentation cases and $5.66 \%$ in reconstruction cases for BellaGel breast implants. The capsular contracture rate was $0.8 \%$ for augmentation and $2.2 \%$ for reconstruction in a 2-year study conducted with Mentor Contour Profile Gel implants [6]. The capsular contracture rate was $8.1 \%$ in augmentation and $8.3 \%$ in reconstruction for Mentor MemoryGel implants in a 3-year study [7]. The capsular contracture rate was $1.9 \%$ in augmentation and 5.9\% for reconstruction for Allergan Style 410 implants in a 3-year study [8]. The capsular contracture rate was $8.8 \%$ and $13 \%$ in augmentation for Mentor and Allergan, respectively, in a 4-year study [9]. The capsular contracture rate of BellaGel breast implants was comparable to or even lower than other breast implants that were observed in a shorter period of time. Overall, the capsular contracture rate of BellaGel breast implants is lower than the value provided in the European guidelines for conformity assessment of breast implants. In the present study, all patients except one were treated with smooth implants. Since it is generally accepted that smooth implants result in a higher capsular contracture rate, a lower rate could have been observed if textured implants had been used for more patients. The capsular contracture rate was higher in reconstruction than in augmentation, which is consistent with the results of previous studies [10]. In this study, the rate of capsular contracture peaked in postoperative year 2 and later decreased. This is consistent with previous studies that showed that capsular contracture is typically an early event that occurs within 2 years of implantation [11].

It is well known that nipple sensitivity decreases after breast augmentation and reconstruction [12]. Our study also showed that the proportion of patients with decreased nipple sensitivity increased immediately after surgery. However, this proportion decreased after approximately 15 months. Among augmentation cases, the proportion of patients with sensory deficits increased again after 48 months. This contradicts previous studies that found that the sensory deficits that occur after breast augmentation recover over time. The authors of the present study were unable to determine the underlying etiology for this pattern.

Overall, the rates of implant rupture and capsular contracture of BellaGel breast implants were comparable to those of other breast implants, even though the period of observation was longer than other studies. Other minor complications are outlined in the publication of the 4-year results [13]. In the present 6-year follow-up report, two additional instances of device breakage were observed. Importantly, however, 4 additional years of follow-up data must be obtained in this study to confirm the safety and effectiveness of BellaGel breast implants.

Despite the 4 additional years of evaluation required, the results found in the first 6 years suggest that the CoSBI manufactured by Hans Biomed is an effective and safe medical device that can be used in breast reconstruction and augmentation. The study did not collect any data regarding breast implant-associated anaplastic large cell lymphoma (BIA-ALCL). As the number of cases of BIA-ALCL are rising, spurring consequent concerns, a future study that evaluates the potential association between BellaGel breast implants and BIA-ALCL is recommended.

\section{NOTES}

\section{Conflict of interest}

$\mathrm{CYH}$ is a consultant for Hans Biomed, which provided funding for the study. SIB received funding for this study. For the remaining authors, no disclosures were provided. Data regarding 
shell properties, the manufacturing process, and the tensile strength of the BellaGel breast implant shell were provided by Hans Biomed.

\section{Ethical approval}

The study was approved by the Institutional Review Board of Seoul National University Bundang Hospital (IRB No. E-100/ 049-001) and Institutional Review Board of Samsung Medical Center (IRB No. SMC 2008-09-073) and performed in accordance with the principles of the Declaration of Helsinki. Written informed consents were obtained.

\section{Author contribution}

Conceptualization: JH Jeong, SI Bang, CY Heo. Data curation: JH Jeong, SI Bang, CY Heo. Formal analysis: JH Jeong, SI Bang, CY Heo. Funding acquisition: SI Bang. Methodology: A Kim, SI Bang, CY Heo. Project administration: SI Bang, CY Heo. Visualization: SI Bang. Writing - original draft: JS Oh. Writing - review \& editing: JS Oh, Y Myung, J Oh, SH Kang, E Park, CY Heo.

\section{ORCID}

$\begin{array}{ll}\text { Joon Seok Oh } & \text { https://orcid.org/0000-0002-8815-9555 } \\ \text { Jae Hoon Jeong } & \text { https://orcid.org/0000-0002-4642-5560 } \\ \text { Yujin Myung } & \text { https://orcid.org/0000-0001-5051-2440 } \\ \text { Jeongseok Oh } & \text { https://orcid.org/0000-0003-2001-084X } \\ \text { Shin Hyeok Kang } & \text { https://orcid.org/0000-0001-9868-2019 } \\ \text { Eonju Park } & \text { https://orcid.org/0000-0002-1516-3078 } \\ \text { Ara Kim } & \text { https://orcid.org/0000-0002-5670-7043 } \\ \text { Sa Ik Bang } & \text { https://orcid.org/0000-0001-5487-8300 } \\ \text { Chan Yeong Heo } & \text { https://orcid.org/0000-0001-9003-7365 }\end{array}$

\section{REFERENCES}

1. American Society of Plastic Surgeons (ASPS). 2018 Plastic surgery statistics report [internet]. Arlington Heights, IL: ASPS; c2019 [cited 2020 Feb 15]. Available from: https:// www.plasticsurgery.org/news/plastic-surgery-statistics.

2. International Society of Aesthetic Plastic Surgery (ISAPS). International survey of aesthetic/cosmetic procedures performed in 2018 [internet]. Hanover, NH: ISAPS [cited $2020 \mathrm{Feb} 15]$. Available from: http://www.isaps.org.
3. Cronin TD, Gerow FJ. Augmentation mammaplasty: a new "natural feel" prosthesis. In: Broadbent TR, editor. Transactions of the Third International Congress of Plastic Surgery. Amsterdam: Excerpta Medica Foundation; 1964. p. 41-9.

4. Maxwell GP, Baker MR. Augmentation mammaplasty: general considerations. In: Spear SL, editor. Surgery of the breast. 2nd ed. Baltimore: Lippincott Williams \& Wilkins; 2006. p. 1237.

5. Danino AM, Basmacioglu P, Saito S, et al. Comparison of the capsular response to the Biocell RTV and Mentor 1600 Siltex breast implant surface texturing: a scanning electron microscopic study. Plast Reconstr Surg 2001;108:2047-52.

6. Cunningham B. The Mentor study on contour profile gel silicone MemoryGel breast implants. Plast Reconstr Surg 2007;120(7 Suppl 1):33S-39S.

7. Cunningham B. The Mentor core study on silicone MemoryGel breast implants. Plast Reconstr Surg 2007;120(7 Suppl 1):19S-32S.

8. Bengtson BP, Van Natta BW, Murphy DK, et al. Style 410 highly cohesive silicone breast implant core study results at 3 years. Plast Reconstr Surg 2007; 120(7 Suppl 1):40S-48S.

9. Stevens WG, Harrington J, Alizadeh K, et al. Five-year follow-up data from the U.S. clinical trial for Sientra's U.S. Food and Drug Administration-approved Silimed ${ }^{\circledast}$ brand round and shaped implants with high-strength silicone gel. Plast Reconstr Surg 2012;130:973-81.

10. Handel N, Jensen JA, Black Q, et al. The fate of breast implants: a critical analysis of complications and outcomes. Plast Reconstr Surg 1995;96:1521-33.

11. Salzberg CA, Ashikari AY, Berry C, et al. Acellular dermal matrix-assisted direct-to-implant breast reconstruction and capsular contracture: a 13-year experience. Plast Reconstr Surg 2016;138:329-37.

12. Banbury J, Yetman R, Lucas A, et al. Prospective analysis of the outcome of subpectoral breast augmentation: sensory changes, muscle function, and body image. Plast Reconstr Surg 2004;113:701-11.

13. Han J, Jeong JH, Bang SI, et al. BellaGel breast implant: 4-year results of a prospective cohort study. J Plast Surg Hand Surg 2019;53:232-9. 